\title{
Public transport: case studies and applications
}

\author{
C. Fleurent • S. Voß
}

Published online: 13 December 2011

(C) Springer-Verlag 2011

As it is stated in the aims and scope of the journal, we aim to present all kinds of research in the area of Public Transport: Planning and Operations. The goal is not only to advance the state of the art but also to advance the state of the practice. "No systems, no impact!" (Nievergelt 1994) Only if we are able to incorporate our ideas and algorithms in computer-aided systems in practice, we have a real impact. This refers to all kinds of systems, whether they be planning and scheduling systems or information systems. That is, besides theoretical papers, the journal also publishes case studies and applications. In that respect we not only aim to serve academia but also an audience of transport operators and consulting firms involved in development and utilization or research of computer-aided planning and scheduling in public transport.

Transit companies have included operations research methods in their planning process for decades. Because of their immediate impact in reducing operating costs, vehicle and crew scheduling optimization algorithms were the first to be practically implemented on a wide-scale basis. The initial models have since evolved in their complexity, and typically integrate both the vehicle and crew scheduling components of the problems. These models, combined with algorithmic advances, can lead to significant savings in practice (see Holmes 2011 for a recent example).

Charles Fleurent is Senior Editor.

Stefan Voß is Editor in Chief.

C. Fleurent

Optimisation and Algorithms, GIRO Inc., 75 Port-Royal St. East, Suite 500, Montréal, Quebec, Canada H3L 3T1

e-mail: charles.fleurent@giro.ca

S. Voß $(\bowtie)$

Institute of Information Systems, University of Hamburg, Von-Melle-Park 5, 20146 Hamburg,

Germany

e-mail: stefan.voss@uni-hamburg.de 
While these traditional scheduling applications continue to progress, more and more public transport companies are applying operations research tools to areas that present new opportunities for efficiencies. For instance, the recent practical availability of data from AVL (Automatic Vehicle Location) and APC (Automatic Passenger Counters) systems makes it feasible to consider new information in the planning processes. In the city of Barcelona, for instance, bus run times are now optimized according to sophisticated criteria that adjust the allocated times to each route segment according to the variance observed over a sample of historical data (see Salicru et al. 2011). On another front, timetables that were once difficult to change after they were established can now be examined and improved. From the Netherlands, NS Railways was awarded with the INFORMS Franz Edelman first prize for their work in this area (see, e.g., Kroon et al. 2009 and Hartog et al. 2009). In Australia, Southern Queensland's integrated transport authority recently engaged in a complete revision of their timetables that led to increased connectivity for passenger transfers, while minimizing operating costs (see Goeldner 2011). Operations research models implemented in the NetPlan module from the HASTUS software suite were at the heart of this effort.

There are also several other areas where new models and algorithms together with advances in information technology and information systems are helping to achieve a better assignment of resources in the public transit domain. In several European companies, work assignment can now consider the personal preferences of individual drivers, leading to a less stressful work environment. Vehicle dispatching can also be optimized according to several criteria that may, for instance, include passenger capacity requirements and distance balancing among the fleet.

Advances in technological developments and information technology such as Radio Frequency Identification (RFID), give an almost continuous rise of new possibilities for innovation in public mass transit. Reasonable uses may especially be envisaged in the form of smart cards, registered smart or mobile phones, and other means of modern access media in public mass transit. Also the discussion to which extent e-ticketing can provide reasonable means to increase the attractiveness of public mass transit is of interest. And, last not least, passenger information can be advanced and is part of public transport products.

This issue presents different case studies and applications in public transport. In their paper "Identifying commuter preferences for existing modes and a proposed Metro in Sydney, Australia with special reference to crowding" D.A. Hensher, J.M. Rose and A.T. Collins provide another Australian experience. As part of a feasibility study to identify the patronage potential of a new metro rail system for Sydney, a modal choice study was undertaken to investigate the role of various attributes and factors and its consequences when dimensioning a new mass transit system. The paper "Solving large scale crew scheduling problems in practice" by E.J.W. Abbink, L. Albino, T. Dollevoet, D. Huisman, J. Roussado and R.L. Saldanha extends the above mentioned Dutch experience at NS. Dealing with large-scale crew scheduling problems, problem decomposition is an issue to be dealth with. However, as the authors show, algorithmic developments may now also try to handle large instances without splitting by using modern operations research techniques. Finally, a third paper is included in this issue by H. Poorzahedy and F. Safari, titled "An ant system application to the bus network design problem: an algorithm and a case study." In 
their study the authors deal with the problem of choosing a subset of interconnected bus routes from among a given set of routes, while minimizing total travel time of the users of the network. One of the simple and yet effective recent metaheuristics, the ant system or ant colony optimization, is applied to design, among others, a bus network using data from the city of Mashhad, Iran. Two of the three papers are provided with extensive additional online material.

In summary, public transport presents several very interesting and challenging problems. Fundamental research thus remains essential to continue moving forward. However, it is only when this research is applied in practice to improve service and efficiently use the scarce resources available that we have really reached our goal.

\section{References}

Goeldner D (2011) Translink Timetable Revamp, Australasian Bus and Coach (May 2011), 34-36

Hartog A, Huisman D, Abbink EJW, Kroon LG (2009) Decision support for crew rostering at NS. Public Transp 1:121-133

Holmes M (2011) HASTUS implementation at MTA Bus Company. In: APTA multimodal operations planning workshop, Seattle, August

Kroon L, Huisman D, Abbink E, Fioole P-J, Fischetti M, Maróti G, Schrijver A, Steenbeek A, Ybema R (2009) The new Dutch timetable: the OR revolution. Interfaces 39(1):6-17

Nievergelt J (1994) Complexity, algorithms, programs, systems: the shifting focus. J Symb Comput 17:297-310

Salicru M, Fleurent C, Armengol JM (2011) Timetable-based operation in urban transport: run-time optimisation and improvements in the operating process. Transp Res. Part A 45:721-740 